\title{
Surveillance des éclosions de maladies entériques en Colombie-Britannique de
} 2009 à 2013

\author{
Taylor $\mathrm{M}^{1^{*}}$, Galanis $\mathrm{E}^{1,2}$,
}

Groupe de travail sur le résumé des éclosions de maladies entériques en Colombie-Britannique : Forsting $\mathrm{S}^{3}$, Gustafson $\mathrm{L}^{4}$, Ip $\mathrm{J}^{3}$, Jeyes $\mathrm{J}^{5}$, Lem $\mathrm{M}^{4}$, Murti $\mathrm{M}^{4}$, Nowakowski $\mathrm{C}^{6}$, Ritson $\mathrm{M}^{3}$, Stone $\mathrm{J}^{4}$, Tone $\mathrm{G}^{7}$

${ }^{1}$ Centre de contrôle des maladies de la Colombie-Britannique, Vancouver (Colombie-Britannique)

${ }^{2}$ École de santé publique et de santé des populations, Université de la Colombie-Britannique, Vancouver

(Colombie-Britannique)

${ }^{3}$ Vancouver Coastal Health Authority, Vancouver (Colombie-Britannique)

${ }^{4}$ Fraser Health Authority, Surrey (Colombie-Britannique)

${ }^{5}$ Interior Health Authority, Vernon (Colombie-Britannique)

${ }^{6}$ Vancouver Island Health Authority, Victoria (Colombie-Britannique)

${ }^{7}$ Northern Health Authority, Prince George (Colombie-Britannique)

* Correspondance : marsha.taylor@bccdc.ca

\section{Résumé}

Contexte : La connaissance des sources d'éclosion de maladies entériques, du fardeau de la maladie, du mode de transmission et de l'utilisation des interventions éclaire la planification, l'élaboration des politiques et les programmes de prévention.

Objectif : Décrire les tendances en matière d'éclosions de maladies entériques qui ont fait l'objet d'enquêtes en Colombie-Britannique entre 2009 et 2013.

Méthodologie : On a effectué une analyse des éclosions de maladies entériques entrées dans un système de déclaration des éclosions en ligne national et sécurisé au moyen du Réseau canadien de renseignements sur la santé publique (RCRSP) et ayant fait l'objet d'une enquête en Colombie-Britannique entre le $1^{\mathrm{er}}$ janvier 2009 et le 31 décembre 2013. Les données comprenaient des renseignements sur l'agent pathogène, le nombre de cas, les hospitalisations, les décès, le contexte, le mode de transmission, la source, les facteurs qui ont contribué à l'éclosion et les interventions. Les éclosions d'infection virale dans des établissements résidentiels et les éclosions associées aux voyages internationaux ont été exclues.

Résultats : On a enquêté sur 104 éclosions en Colombie-Britannique entre 2009 et 2013. Parmi celles-ci, 93 ont été déclarées par des organisations de la Colombie-Britannique, et 11 ont été déclarées par l'Agence de la santé publique du Canada (ASPC) et ont fait l'objet d'une enquête nationale. Il y a eu 21 éclosions par année en moyenne. Dans l'ensemble, le taux annuel de vagues d'intoxication alimentaire en ColombieBritannique était de 2,8 par million de personnes. On a identifié un agent pathogène, notamment les norovirus, la Salmonella et l'infection à E. coli., dans (76\%) des éclosions. II y a eu 108 hospitalisations ( $3,8 \%$ de tous les cas) et deux décès $(0,1 \%$ de tous les cas), le premier causé par le botulisme et le second par une infection à $E$. coli 0157 . Les établissements de services alimentaires étaient le milieu le plus souvent signalé $(33,7 \%)$ et étaient suivis de la collectivité $(24 \%)$ et des réceptions privées (12,5\%). Les types d'aliments les plus souvent signalés étaient les fruits et légumes, la viande et les fruits de mer. Les données montraient une combinaison agent pathogène-source alimentaire entre la Salmonella et les œufs.

Conclusion : Il s'agit de la première publication dans laquelle on résume les tendances en matière d'éclosions de maladies entériques en Colombie-Britannique, ce qui comprend l'évaluation des sources, du fardeau et des interventions. La déclaration et l'analyse continues des données sur les éclosions en Colombie-Britannique permettront d'améliorer l'évaluation des tendances dans les sources et les agents pathogènes au fil du temps et d'approfondir les connaissances relatives à l'efficacité des interventions associées aux éclosions. 


\section{Introduction}

On estime à 552209 le nombre de cas de maladies d'origine alimentaire contractées au Canada en Colombie-Britannique chaque année (données non publiées, Centre de contrôle des maladies de la Colombie-Britannique et Agence Santé Publique Canada, 2014). Malgré le fait qu'une petite partie de ces cas soit associée à des éclosions confirmées (entre $0,8 \%$ et $2,5 \%$ de tous les cas) (1), les éclosions sont une précieuse source d'information sur les sources de maladie, le fardeau de la maladie, les modes de transmission et les interventions (2). Les autorités de santé publique, les décideurs, les professionnels de la salubrité des aliments et l'industrie alimentaire peuvent se servir de cette information pour fixer leurs priorités et ainsi que planifier et mettre en œuvre des programmes de prévention. Le gouvernement de la Colombie-Britannique a mis en œuvre un programme de surveillance des éclosions de maladies entériques en 2008 afin de décrire les tendances, d'améliorer la connaissance, de déterminer la source et d'évaluer les interventions et l'utilisation des ressources.

L'objectif de l'étude consiste à décrire les tendances en matière d'éclosions de maladies entériques ayant fait l'objet d'une enquête en Colombie-Britannique entre 2009 et 2013, ainsi qu'à déterminer les sources et à décrire les interventions.

\section{Méthodologie}

Les épidémies de gastro-entérite doivent être déclarées en Colombie-Britannique (3). En août 2008, on a mis en ligne, en Colombie-Britannique, un système national de déclaration des éclosions au moyen du Réseau canadien de renseignements sur la santé publique. En Colombie-Britannique, les éclosions de maladies entériques sont entrées dans ce système par les autorités sanitaires locales et le Centre de contrôle des maladies de la Colombie-Britannique. Le système du Réseau est également utilisé par d'autres provinces et territoires du Canada.

Il y a deux types d'éclosions de maladies gastro-intestinales dans le système, soit l'éclosion au sein de la collectivité, où au moins deux cas non liés de maladies similaires pouvant être liés entre eux sur le plan épidémiologique (c'est-à-dire être liés en fonction du moment du lieu ou du type d'exposition), et l'éclosion dans un établissement, où au moins trois cas de maladies similaires peuvent être liés entre eux sur le plan épidémiologique (c'est-à-dire être liés en fonction d'une exposition dans une période de quatre jours dans un milieu institutionnel).

Toutes les données sont saisies à la main (rétrospectivement) et comprennent des renseignements sur les agents pathogènes, le nombre de cas, les hospitalisations, les décès, le contexte, le mode de transmission, la source, les facteurs qui ont contribué à l'éclosion et les interventions. Chaque autorité sanitaire locale est tenue d'entrer dans le système les éclosions ayant fait l'objet d'une enquête sur leur territoire. Le Centre de contrôle des maladies de la Colombie-Britannique entre les éclosions multirégionales, et l'Agence entre les éclosions multiprovinciales et multiterritoriales.

Le présent rapport comprend des données sur les enquêtes sur les éclosions de maladies entériques déclarées en Colombie-Britannique entre 2009 et 2013. Les éclosions nationales (déclarées par l'Agence de la santé publique du Canada) qui comprenaient les cas relevés en Colombie-Britannique ont également été incluses. Les éclosions d'infection virale dans des établissements résidentiels et les éclosions associées aux voyages internationaux ont été exclues.

Les données ont été extraites le 6 mars 2014. Les données relatives aux éclosions nationales ont quant à elles été extraites le 5 juin 2014. On a comparé le milieu, les facteurs contributifs et les interventions relatifs aux éclosions déclarées comme étant d'origine alimentaire et à la transmission interhumaine. On ne s'est appuyé que sur les éclosions déclarées comme étant d'origine alimentaire pour l'attribution des sources. On a lié les éclosions aux manipulateurs d'aliments lorsqu'un agent pathogène était découvert chez un manipulateur d'aliment ou quand on découvrait un manipulateur d'aliment infecté. L'année et le mois de l'enquête sur l'éclosion étaient fondés sur la date de commencement de l'enquête. On a calculé la durée des éclosions au moyen des dates d'apparition et de disparition des symptômes indiquées. 


\section{Résultats}

On a enquêté sur 104 éclosions en Colombie-Britannique entre 2009 et 2013. Les organisations de la Colombie-Britannique ont déclaré 93 éclosions, et l'Agence de la santé publique du Canada a déclaré 11 éclosions. On a enquêté sur une moyenne de 21 éclosions, une médiane de 22 éclosions et un écart de 16 à 26 éclosions par année (Figure 1). On a noté une augmentation d'environ $40 \%$ dans les éclosions ayant fait l'objet d'une enquête en 2011, 2012 et 2013. Le taux annuel de vagues d'intoxication alimentaire en Colombie-Britannique était de 2,8 par million de personnes.

Figure 1 : Nombre d'éclosions de maladies entériques déclarées par année en Colombie-Britannique ( $N$ = 104)

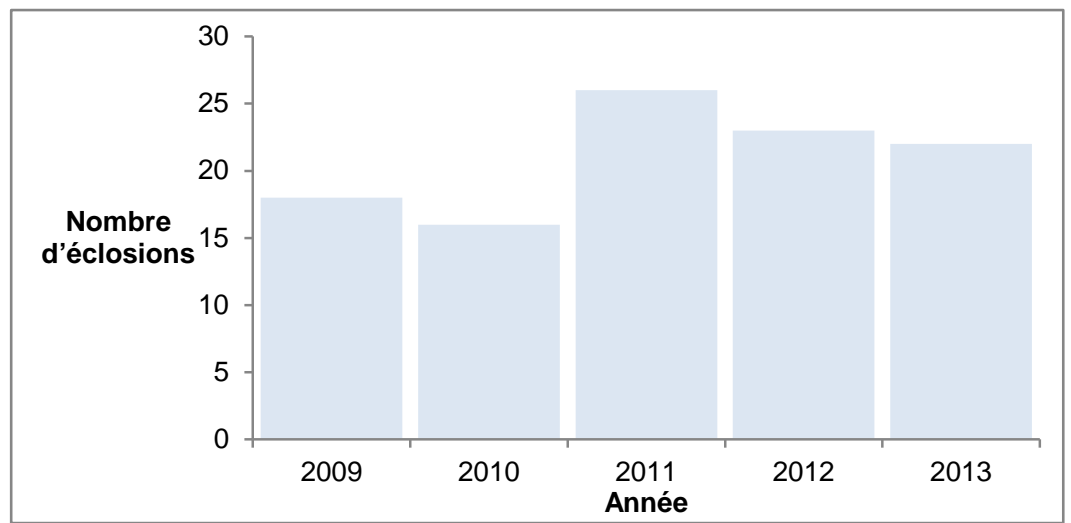

On a déclaré 50 (48,1\%) éclosions bactériennes et $42(40,4 \%)$ éclosions virales. On a identifié en laboratoire un agent pathogène pour $79(76 \%)$ des éclosions (Tableau 1). Les agents pathogènes les plus fréquemment déclarés étaient les norovirus, la Salmonella et l'infection à $E$. coli (Tableau 2). Enteritidis était le sérotype de Salmonella $(n=13,50 \%)$, et toutes les infections à l'E. coli étaient causées par l'E. coli O157.

Il y avait un total de 2134 cas liés à l'éclosion (cas cliniques et cas confirmés en laboratoire) (Tableau 1). La majorité des cas $(76,4 \%)$ ont été identifiés de manière clinique, et les éclosions causées par des agents pathogènes viraux étaient les plus nombreuses et représentaient la majorité des cas cliniques. Une hospitalisation a été nécessaire dans 108 (5,1\%) cas. La majorité des hospitalisations $(81,75 \%)$ étaient dues aux éclosions causées par une bactérie. Les agents pathogènes à l'origine de la plupart des hospitalisations étaient la Salmonella (38, 35,2 \%), l'E. coli (37, 34,3\%) et les norovirus (10, 9,3\%). Les infections bactériennes ont causé deux décès (Tableau 1), dont un par le botulisme et l'autre par une infection à l'E. coli O157.

Il y avait une moyenne de 20,3 cas par éclosions. Les éclosions causées par des agents pathogènes viraux étaient les plus nombreuses (29 cas), et celles causées par les levures et les champignons étaient les moins nombreuses (7 cas). La médiane de la durée des éclosions était de quatre jours. Les éclosions causées par des bactéries et des parasites étaient considérablement plus longues que les autres (durées de 11 et 16 jours respectivement) (Tableau 1). 
Tableau 1 : Les caractéristiques des enquêtes sur les éclosions de maladies entériques par type d'agent pathogène, Colombie-Britannique, 2009 à 2013

\begin{tabular}{|c|c|c|c|c|c|c|c|}
\hline Caractéristiques & $\begin{array}{l}\text { Éclosion } \\
\text { bactérienne } \\
(n=50)\end{array}$ & $\begin{array}{l}\text { Éclosion } \\
\text { virale } \\
n=42\end{array}$ & $\begin{array}{l}\text { Éclosions } \\
\text { parasites } \\
(n=2)\end{array}$ & $\begin{array}{l}\text { Éclosions } \\
\text { d'origine } \\
\text { inconnue } \\
(n=4)\end{array}$ & $\begin{array}{l}\text { Cas liés à } \\
\text { une toxine } \\
\text { ou à un } \\
\text { produit } \\
\text { chimique } \\
(n=5)\end{array}$ & $\begin{array}{l}\text { Cas liés à des } \\
\text { levures ou } \\
\text { des } \\
\text { champignons } \\
n=1\end{array}$ & $\begin{array}{c}\text { Total } \\
(n=104)\end{array}$ \\
\hline $\begin{array}{l}\text { Nombre (\%) d'éclosions } \\
\text { confirmées en } \\
\text { laboratoire }\end{array}$ & $\begin{array}{c}49 \\
(98 \%)\end{array}$ & $\begin{array}{c}23 \\
(54,8 \%)\end{array}$ & $\begin{array}{c}2 \\
(100 \%)\end{array}$ & $\begin{array}{c}0 \\
(0 \%)\end{array}$ & $\begin{array}{c}5 \\
100 \%\end{array}$ & $\begin{array}{c}0 \\
(0 \%)\end{array}$ & $79(76 \%)$ \\
\hline $\begin{array}{l}\text { Nombre total de cas } \\
\text { confirmés en laboratoire }\end{array}$ & 398 & 80 & 12 & 0 & 12 & 0 & 502 \\
\hline $\begin{array}{l}\text { Nombre total de cas } \\
\text { cliniques }\end{array}$ & 351 & 1138 & 16 & 45 & 75 & 7 & 1632 \\
\hline $\begin{array}{l}\text { Nombre moyen de } \\
\text { cas }^{1 /} \text { ou d'éclosions }\end{array}$ & 14,9 & 29,0 & 22,0 & 11,3 & 15,4 & 7,0 & 20,3 \\
\hline $\begin{array}{l}\text { Nombre total et } \% \text { de } \\
\text { taux d'hospitalisation }\end{array}$ & $81(75 \%)$ & $20(25 \%)$ & 0 & 0 & 7 & 0 & 108 \\
\hline $\begin{array}{l}\text { Nombre total et } \% \text { de } \\
\text { taux de décès }\end{array}$ & $2(100 \%)$ & 0 & 0 & 0 & 0 & 0 & 2 \\
\hline $\begin{array}{l}\text { Durée médiane (nombre } \\
\text { de jours) de l'éclosion en } \\
\text { fonction de la date } \\
\text { d'apparition }\end{array}$ & $\begin{array}{c}11 \\
(0 \text { à 234) }\end{array}$ & $\begin{array}{c}4 \\
\text { (0 à 137) }\end{array}$ & 16 & $\begin{array}{l}1 \\
(0 \text { à } 2)\end{array}$ & $\begin{array}{c}0 \\
(0 \text { à 11) }\end{array}$ & 0 & $\begin{array}{c}4 \\
\text { (0 à 234) }\end{array}$ \\
\hline
\end{tabular}

${ }^{1}$ Comprend les cas cliniques et les cas confirmés en laboratoire.

Tableau 2 : Éclosions de maladies entériques par agent pathogène en Colombie-Britannique de 2009 à 2013

\begin{tabular}{|c|c|c|}
\hline Agent pathogène & Nombre (\%) & \\
\hline Norovirus & $38(36,5 \%)$ & \\
\hline Salmonella & $26(25 \%)$ & \\
\hline Escherichia coli & $12(11,5 \%)$ & \\
\hline Clostridium botulinum & $3(2,9 \%)$ & \\
\hline Campylobactérie & $2(1,9 \%)$ & \\
\hline Hépatite A & $2(1,9 \%)$ & \\
\hline Intoxication à l'histamine & $2(1,9 \%)$ & \\
\hline Intoxication par des mollusques ou crustacés ${ }^{1}$ & $2(1,9 \%)$ & \\
\hline Staphylococcus & $2(1,9 \%)$ & \\
\hline Autre $^{2}$ & $7(6,7 \%)$ & \\
\hline Éclosions d'origine inconnue $^{3}$ & $8(7,7 \%)$ & \\
\hline Total & 104 & \\
\hline \multicolumn{3}{|c|}{$\begin{array}{l}\text { Intoxication par des mollusques ou crustacés (1), intoxication par phycotoxine diarrhéique (1). } \\
\text { Aeromonas (1), toxine ou produit chimique (1), Cryptosporidium (1), Cyclospora (1), Shigella (1), Clostridium perfrigens (1), Clostridium difficile } \\
\text { (1). } \\
\text { Ce chiffre comprend une éclosion bactérienne indéterminée, deux éclosions virales indéterminées et une infection aux levures ou aux champignons } \\
\text { indéterminée, ainsi que quatre éclosions dont l'agent pathogène en cause n’a pu être déterminé. Dans le Tableau 2, quatre des éclosions sont } \\
\text { caractérisées dans leurs groupes de niveau supérieur. }\end{array}$} \\
\hline
\end{tabular}

L'exposition alimentaire était le mode de transmission prévalent (59,6 \%) (Tableau 3). Parmi les 62 éclosions d'origine alimentaire, 40 (64,5\%) étaient causées par une bactérie. La cause prévalente était la Salmonella $(n=22)$. Parmi les éclosions résultant d'une transmission interhumaine, $22(95,7 \%)$ étaient causées par des norovirus.

Les établissements de services alimentaires étaient le milieu le plus souvent signalé $(33,7 \%)$, suivis de la collectivité (24\%) et des réceptions privées (12,5\%) (Tableau 3). Les éclosions dans des établissements de 
services alimentaires étaient causées par des aliments et la transmission interhumaine. Parmi les huit cas d'éclosions résultant d'une transmission interhumaine dans les établissements de services alimentaires, deux étaient attribuables à des manipulateurs d'aliments malades. Les éclosions résultant d'une transmission interhumaine étaient prévalentes dans les installations, comme les hôpitaux, les écoles et les hôtels (Tableau 3).

Tableau 3 : Éclosions de maladies entériques par mode de transmission et milieu, ColombieBritannique, de 2009 à 2013

\begin{tabular}{|l|c|c|c|c|c|c|}
\hline $\begin{array}{l}\text { Milieu } \\
\text { d'éclosion }\end{array}$ & $\begin{array}{c}\text { Origine } \\
\text { alimentaire }\end{array}$ & $\begin{array}{c}\text { Transmission } \\
\text { interhumaine }\end{array}$ & $\begin{array}{c}\text { Origine } \\
\text { inconnue }\end{array}$ & Autre & Eau & Total \\
\hline $\begin{array}{l}\text { Établissement } \\
\text { de services } \\
\text { alimentaires }\end{array}$ & $24(38,7 \%)$ & $8(34,8 \%)$ & $1(8,3 \%)$ & $2(25 \%)$ & $0(0 \%)$ & $35(33,7 \%)$ \\
\hline Collectivité & $17(27,4 \%)$ & $2(9 \%)$ & $5(41,7 \%)$ & $\begin{array}{c}1 \\
(12,5 \%)\end{array}$ & $0(0 \%)$ & $25(24 \%)$ \\
\hline $\begin{array}{l}\text { Réception } \\
\text { privée }\end{array}$ & $10(16,1 \%)$ & $3(13 \%)$ & $0(0 \%)$ & $0(0 \%)$ & $0(0 \%)$ & $13(12,5 \%)$ \\
\hline Établissement ${ }^{2}$ & $5(8,1 \%)$ & $4(17,4 \%)$ & $1(8,3 \%)$ & $\begin{array}{c}1 \\
(12,5 \%)\end{array}$ & $0(0 \%)$ & $11(10,6 \%)$ \\
\hline $\begin{array}{l}\text { Installation ne } \\
\text { faisant pas } \\
\text { partie d'un } \\
\text { établissement }\end{array}$ & $0(0 \%)$ & $3(13 \%)$ & $1(8,3 \%)$ & $0(0 \%)$ & $0(0 \%)$ & $4(3,8 \%)$ \\
\hline $\begin{array}{l}\text { Installation de } \\
\text { loisir }\end{array}$ & $0(0 \%)$ & $2(8,7 \%)$ & $0(0 \%)$ & $\begin{array}{c}1 \\
(12,5 \%)\end{array}$ & $0(0 \%)$ & $3(2,9 \%)$ \\
\hline Plus d'un milieu & $3(4,8 \%)$ & $1(4,3 \%)$ & $1(8,3 \%)$ & $\begin{array}{c}1 \\
(12,5 \%)\end{array}$ & $0(0 \%)$ & $6(5,8 \%)$ \\
\hline Autre & $2(3,2 \%)$ & $0(0 \%)$ & $2(16,6 \%)$ & $2(25 \%)$ & $0(0 \%)$ & $6(5,8 \%)$ \\
\hline Inconnu & $1(1,6 \%)$ & $0(0 \%)$ & $0(0 \%)$ & $0(0 \%)$ & $0(0 \%)$ & $1(1 \%)$ \\
\hline Total & $62(59,6 \%)$ & $23(22,1 \%)$ & $11(10,6 \%)$ & $8(7,7 \%)$ & $0(0 \%)$ & 104 \\
\hline
\end{tabular}

${ }^{1}$ Inclut la transmission d'un animal à une personne, la transmission environnementale, les autres types de transmission et les modes de transmission multiples.

${ }^{2}$ Comprend les cas résidentiels et non résidentiels.

${ }^{3}$ Exemples : écoles, hôtels et hôpitaux.

On a déterminé que la source de 45 (72,6 \%) des éclosions d'origine alimentaire (Tableau 4) était des aliments et les plus souvent signalés étaient les fruits et légumes, la viande et les fruits de mer. On a trouvé le plus grand nombre d'agents pathogènes $(n=5)$ dans les fruits et les légumes qui incluaient des fruits et des légumes frais, congelés et en conserve. Parmi les 14 éclosions de Salmonella dont on a déterminé la source, cinq des éclosions signalées étaient principalement à des œufs (35,7\%). La seule éclosion attribuable à des produits laitiers a été causée par du fromage non pasteurisé. Les norovirus ont causé dix éclosions d'origine alimentaire. On a déterminé la source de neuf d'entre elles. Les éclosions de norovirus étaient attribuables à des fruits de mer, des aliments mixtes, des fruits et des légumes. On a déterminé qu'un manipulateur d'aliments infecté avait joué un rôle dans les huit éclosions de source déterminée et dans l'éclosion de source indéterminée. Dans huit des éclosions avec source et dans l'éclosion sans source, on a déterminé qu'un manipulateur d'aliments était le facteur contributif (les données non montrées). 
Tableau 4 : Éclosions d'origine alimentaire par agent pathogène et source, Colombie-Britannique, de 2009 de 2013

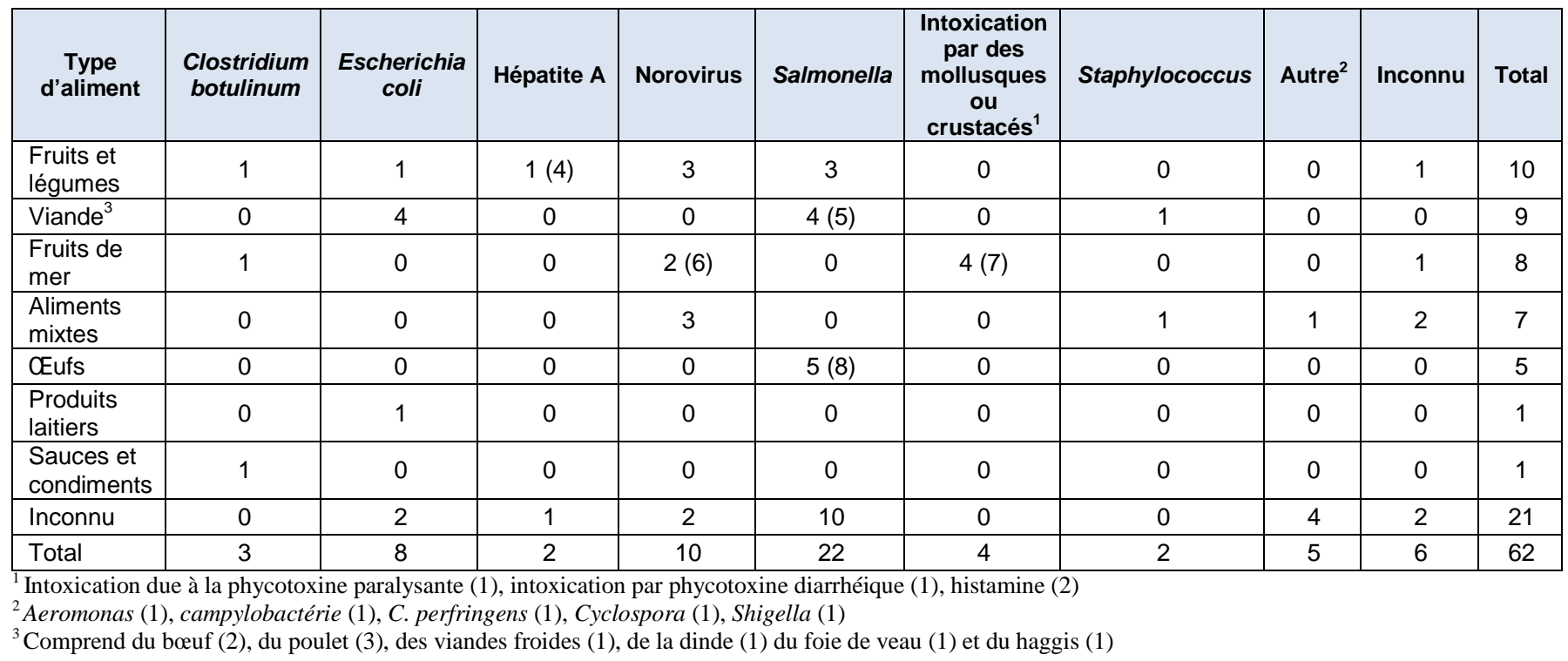

Les œufs étaient à l'origine du plus grand nombre de cas $(n=196)$, aliments mixtes $(n=168)$ et fruits de $\operatorname{mer}(n=139)$. Ces aliments étaient à l'origine de $45,5 \%$ de tous les cas d'éclosions d'origine alimentaire.

Les facteurs contributifs prévalents des éclosions d'origine alimentaire étaient associés aux processus de production alimentaire (p. ex., échecs à des points critiques, cuisson inadéquate et contamination croisée). Dans le cas des éclosions résultant d'une transmission interhumaine, les facteurs contributifs prévalents étaient l'exposition à une personne malade, à un cas ou à un environnement contaminé (Tableau 5).

Tableau 5 : Facteurs contributifs des éclosions d'origine alimentaire et des éclosions résultant d'une transmission interhumaine, Colombie-Britannique, de 2009 à 2013

\begin{tabular}{|c|c|c|}
\hline Facteurs contributifs & $\begin{array}{l}\text { Origine alimentaire } \\
(\mathrm{N}=62)\end{array}$ & $\begin{array}{l}\text { Transmission interhumaine } \\
\qquad(\mathrm{N}=23)\end{array}$ \\
\hline Échec de point de contrôle & $17(27,4 \%)$ & $1(4,3 \%)$ \\
\hline Contamination croisée & $11(17,7 \%)$ & $3(13 \%)$ \\
\hline $\begin{array}{l}\text { Température inadéquate (p. ex., réfrigération } \\
\text { ou conservation des aliments chauds) }\end{array}$ & $12(19,4 \%)$ & 0 \\
\hline Réchauffement inadéquat & $1(1,6 \%)$ & 0 \\
\hline Manipulateur d'aliments infecté & $11(17,7 \%)$ & $2(8,7 \%)$ \\
\hline Cuisson inadéquate & $12(19,4 \%)$ & 0 \\
\hline Mauvaise hygiène & $8(12,9 \%)$ & $6(26,1 \%)$ \\
\hline Contact avec un personnel soignant & 0 & $2(8,7 \%)$ \\
\hline Exposition à un cas confirmé ou probable & $1(1,6 \%)$ & $7(30,4 \%)$ \\
\hline Hygiène de l'environnement inadéquate & $5(8 \%)$ & $3(13 \%)$ \\
\hline
\end{tabular}

Les interventions les plus souvent effectuées pour maîtriser les éclosions étaient la sensibilisation, la désinfection des lieux et le regroupement en cohorte des cas et du personnel (Tableau 6). La sensibilisation et la désinfection des lieux constituaient les interventions les plus répandues pour lutter contre les éclosions résultant d'une transmission interhumaine. Les interventions les plus souvent effectuées pour maîtriser les éclosions d'origine alimentaire consistaient à rappeler des produits et à fermer des installations. Dans le cas des éclosions résultant d'une transmission interhumaine, elles consistaient à limiter l'accès, les déplacements et les visites, et à regrouper les cas et le personnel en cohorte. Dans sept cas d'éclosions d'origine alimentaire, on a signalé un changement de politique comme intervention. On a diffusé un 
communiqué pour dix éclosions (neuf d'origine alimentaire et une résultant d'une transmission interhumaine). La sensibilisation était l'intervention la plus répandue pour l'ensemble des sources alimentaires. Les rappels visaient surtout la viande (cinq), les fruits de mer (deux) et les fruits (deux). On a procédé à la désinfection des installations dans le cas d'éclosions causées par huit sources alimentaires différentes.

Tableau 6 : Interventions effectuées pour maîtriser les éclosions d'origine alimentaire et les éclosions résultant d'une transmission interhumaine, Colombie-Britannique, de 2009 à 2013

\begin{tabular}{|l|c|c|c|c|}
\hline \multicolumn{1}{|c|}{ Intervention } & $\begin{array}{c}\text { Origine alimentaire } \\
\mathbf{( N = 6 2 )}\end{array}$ & $\begin{array}{c}\text { Transmission } \\
\text { interhumaine } \\
(\mathbf{N}=\mathbf{2 3})\end{array}$ & $\begin{array}{c}\text { Autre } \\
\mathbf{N = 2 0}\end{array}$ & Total \\
\hline Sensibilisation & $36(59 \%)$ & $15(24,6 \%)$ & $10(16,4 \%)$ & 61 \\
\hline Désinfection de l'installation & $13(40,6 \%)$ & $9(28,1 \%)$ & $10(31,3 \%)$ & 32 \\
\hline $\begin{array}{l}\text { Regroupement des cas ou } \\
\text { du personnel en cohorte }\end{array}$ & $2(11,8 \%)$ & $8(47,1 \%)$ & $7(41,2 \%)$ & 17 \\
\hline Exclusion du personnel & $7(46,7 \%)$ & $5(33,3 \%)$ & $3(20 \%)$ & 15 \\
\hline Rappel & $12(100 \%)$ & 0 & 0 & 12 \\
\hline $\begin{array}{l}\text { Restriction de l'accès à } \\
\text { l'installation, les } \\
\text { déplacements et les visites }\end{array}$ & $2(18,2 \%)$ & $7(63,6 \%)$ & $2(18,2 \%)$ & 11 \\
\hline Communiqué & $9(90 \%)$ & $1(10 \%)$ & 0 & 10 \\
\hline Fermeture de l'installation & $6(66,7 \%)$ & $1(11,1 \%)$ & $2(22,2 \%)$ & 9 \\
\hline Changement stratégique & $7(100 \%)$ & 0 & 0 & 7 \\
\hline $\begin{array}{l}\text { Immunisation des personnes } \\
\text { susceptibles d'être malades }\end{array}$ & $1(100 \%)$ & 0 & 0 & 1 \\
\hline Avis d'ébullition & 0 & 0 & 0 & 0 \\
\hline
\end{tabular}

\section{Discussion}

Au cours de la période de cinq ans, le nombre important d'éclosions de maladies entériques déclarées a imposé un lourd fardeau de maladie à la Colombie-Britannique. L'augmentation du nombre d'éclosions déclarées entre 2011 et 2013 est probablement due aux efforts visant à améliorer la déclaration des éclosions. II faut ajouter à cela la décision de cesser la déclaration des éclosions virales dans les installations résidentielles à partir de juillet 2011, qui pourrait avoir amélioré la déclaration des éclosions associées à d'autres modes de transmission, à d'autres milieux et à d'autres sources. L'exclusion limite la capacité à déterminer le nombre total d'éclosions de maladies entériques causées par toutes les sources et tous les agents pathogènes dans tous les milieux, à l'échelle provinciale.

Les norovirus étaient la cause d'éclosion prévalente, suivis de la Salmonella. Les deux sont parmi les cinq principaux agents pathogènes à l'origine de maladies d'origine alimentaire contractées en milieu résidentiel en Colombie-Britannique (données non publiées, Centre de contrôle des maladies de la Colombie-Britannique et Agence de la santé publique du Canada, 2014). Ces agents pathogènes sont aussi la principale cause d'éclosions aux États-Unis (2). Les éclosions virales comportaient le plus grand nombre de cas, et les éclosions bactériennes étaient la principale cause d'hospitalisations et de décès. Les éclosions bactériennes et parasites, et les éclosions liées à une toxine ou à un produit chimique avaient davantage tendance à être confirmées en laboratoire que les éclosions virales. Cela est probablement attribuable au fait qu'on peut enquêter sur éclosions virales et les gérer sans diagnostic en laboratoire, et que celles-ci sont spontanément résolutives et ne sont pas associées à des cas graves.

Le taux d'hospitalisation $(3,8 \%)$ est comparable à celui des États-Unis $(3,4 \%)$ et inférieur à celui de l'Europe $(13,8 \%)(2,10)$. Le taux de décès $(0,1 \%)$ est inférieur à celui des États-Unis $(0,7 \%)$, mais légèrement supérieur à celui de l'Europe $(0,03 \%)(2,10)$. Aux États-Unis et en Colombie-Britannique, la Salmonella, l'E. coli et les norovirus ont causé le nombre et le taux d'hospitalisations les plus importants. Ces similarités pourraient être dues au fait que les cas les plus graves sont susceptibles de faire l'objet de tests et d'être confirmés en laboratoire dans les deux pays. La différence par rapport au taux 
d'hospitalisation en Europe pourrait être attribuable à l'inclusion de maladies plus graves, comme la toxoplasmose et la tularémie. La différence entre le taux de décès en Colombie-Britannique, aux États-Unis et en Europe pourrait être attribuable à de faibles chiffres.

Le taux annuel d'éclosions d'origine alimentaire en Colombie-Britannique (2,8 par million d'habitants) est inférieur à celui des États-Unis (4,8 par million d'habitants) (2). Cela pourrait être attribuable à la différence des systèmes et des méthodes de déclaration.

Les établissements de services alimentaires étaient le principal milieu associé à toutes les éclosions (34 \%) en Colombie-Britannique. Parmi les éclosions de Salmonella en Colombie-Britannique, les établissements de services alimentaires demeuraient le milieu prévalent et étaient associés à $47,6 \%$ des éclosions. La situation est similaire aux États-Unis, où les restaurants et les épiceries comportant un lieu unique pour la préparation de nourriture étaient le milieu prévalent pour les éclosions d'origine alimentaire, mais dans une proportion plus élevée (68\%). Les exploitations alimentaires commerciales néozélandaises étaient le deuxième milieu en importance pour les éclosions de Salmonella (31\%), alors que les lieux résidentiels étaient le milieu le plus signalé $(2,9)$.

La majorité des éclosions étaient d'origine alimentaire (59,6 \%). La Salmonella était la principale cause d'éclosions d'origine alimentaire (35,8 \%). Parmi les éclosions de Salmonella, 84,6 \% étaient d'origine alimentaire, ce qui est supérieur au taux néozélandais (63\%) (9). La Salmonella étaient également la principale cause d'éclosions d'origine alimentaire déclarées en Europe (10).

On déterminé une source d'exposition pour $72,6 \%$ des éclosions d'origine alimentaire. Il s'agit d'une augmentation par rapport aux déclarations faites en Colombie-Britannique et dans les autres provinces et territoires $(11,12)$. Cela pourrait être attribuable au fait que les éclosions de source indéterminée sont plus susceptibles d'être déclarées que les autres. Les fruits et les légumes frais, la viande et les fruits de mer étaient les aliments les plus souvent en cause (16\%, $15 \%$ et $13 \%$ respectivement). Les fruits et les légumes frais étaient à l'origine de plus d'éclosions que la viande, les œufs et les produits laitiers. Une analyse américaine a révélé que les produits de la viande étaient la principale source d'éclosions d'origine alimentaire et que les légumes-feuilles étaient la cause d'un nombre grandissant d'éclosions (2) au cours des dernières années. Les éclosions causées par des fruits et des légumes frais ont augmenté en Amérique du Nord $(13,14,15)$. La surveillance de ces tendances au moyen des données sur les éclosions permettra de réordonner les priorités en matière d'intervention.

Les données montrent un lieu entre la Salmonella et les œufs. Durant cette période, la Colombie-Britannique a enquêté sur un grand nombre d'éclosions prolongées de Salmonella Enteritidis associées à des œufs (8). Les œufs sont demeurés la principale cause d'éclosions de Salmonella Enteritidis aux États-Unis, mais on a noté une diminution graduelle des éclosions de Salmonella causés par les œufs (2). Les données européennes ont montré que les œufs et les ovoproduits sont le principal véhicule alimentaire pour la Salmonella (10). On a déjà procédé à l'attribution propre aux aliments au Canada, deux fois en s'appuyant sur l'avis d'experts et une fois en s'appuyant sur les données sur les éclosions $(16,17,18)$. Dans les trois cas, on a déterminé que la viande de volaille était la source la plus probable des infections à la Salmonella. On a déterminé que les œufs étaient la deuxième source en importance selon l'avis des experts. Selon les experts, une bonne partie des infections entériques d'origine bactérienne seraient attribuables à des fruits et à des légumes frais, ce qui correspond aux conclusions de la présente étude $(16,17)$.

Les éclosions sont une source validée de données d'attribution. Parmi les forces des données sur les éclosions, mentionnons le lien clair entre les agents pathogènes et les aliments, l'accès aux données au fil du temps et l'inclusion d'une grande variété d'aliments qui ne sont pas nécessairement pris en compte par d'autres méthodes (9). Cependant, les éclosions ne font pas toutes l'objet d'une enquête et ne sont pas toutes déclarées. En outre, les données provenant de tous les systèmes ne sont pas toujours comparables $(19,20)$. 
On a établi que des manipulateurs d'aliments avaient joué un rôle dans neuf éclosions de norovirus. Cela met en évidence la nécessité d'améliorer la sensibilisation et les ressources visant les manipulateurs d'aliments et leur employeur, ainsi que les interventions visant à identifier et à exclure rapidement les travailleurs malades.

La sensibilisation et la désinfection des installations étaient les interventions les plus fréquentes. Les autres interventions, comme les rappels et les changements stratégiques sont moins fréquentes, car elles nécessitent la détermination d'une source alimentaire ou d'un problème particulier. Les interventions sont influencées par le milieu dans lequel elles sont menées. C'est notamment le cas des interventions dans des milieux institutionnels où la transmission interhumaine peut être prévalente. Cependant, il est impossible de dire si les interventions effectuées ont permis d'empêcher de nouveaux cas, car on n'a pu obtenir la date des interventions. La documentation relative à l'efficacité des interventions en réponse aux éclosions est limitée, notamment dans le cas des éclosions généralisées et des éclosions au sein de la collectivité. Une enquête plus approfondie faciliterait la prise de décisions, l'attribution des ressources et les enquêtes sur les éclosions.

L'analyse a montré que le système de surveillance convient à la tâche, malgré ses limites. On se concentre sur un petit nombre d'éclosions déclarées sur une courte période. Par conséquent, il est impossible d'effectuer une analyse plus approfondie par agent pathogène ou au moyen de l'évaluation de l'évolution des tendances. On finira par surmonter cet obstacle quand plus d'éclosions seront déclarées. En outre, les autorités provinciales et territoriales en matière de santé publique doivent entrer les éclosions dans le système pour que celui-ci fonctionne. Bien qu'on ait mis en place des processus pour vérifier l'entrée des éclosions connues, il se pourrait que certaines éclosions ne soient pas détectées et déclarées, et ne fassent pas l'objet d'une enquête. Enfin, les données décentralisées peuvent aussi avoir une incidence sur la qualité des données. Lorsque des problèmes sur le plan de la qualité des données sont notés, on apporte des améliorations aux normes ou au système.

\section{Conclusion}

La surveillance des éclosions de maladies entériques en Colombie-Britannique fournit de l'information sur les tendances, les sources, les milieux et les modes de transmission. On s'est appuyé sur les données pour éclairer les évaluations techniques et les évaluations des risques, les rapports et les publications. On pourrait s'appuyer sur des données supplémentaires pour éclairer l'établissement des priorités locales en matière de salubrité des aliments, pour formuler des messages concernant les combinaisons d'agents pathogènes et d'aliments et pour affecter des ressources à des interventions particulières.

La déclaration et l'analyse continues des données sur les éclosions en Colombie-Britannique permettront d'améliorer l'évaluation des tendances dans les sources et les agents pathogènes au fil du temps et d'approfondir les connaissances relatives à l'efficacité des interventions associées aux éclosions.

\section{Remerciements}

Les auteurs souhaitent remercier leurs collègues au sein de l'autorité sanitaire qui ont saisi les éclosions, ainsi que les laboratoires et le Laboratoire de santé publique et de référence en microbiologie de la Colombie-Britannique pour le soutien au diagnostic.

\section{Conflit d'intérêts}

Aucun.

\section{Financement}

Aucun. 


\section{Références}

(1) Public Health Agency of Canada. Foodnet Canada 2013 Short Report [Internet]. Ottawa ON: PHAC; September 2014. (Disponible en français : http://www.phac-aspc.gc.ca/foodnetcanada/report-rapport-2013-fra.php)

(2) Gould H, Walsh KA, Vieira AR, Herman K, Williams IT, Hall AJ, Cole D. Surveillance for foodborne disease outbreaks-United States, 1998-2008. MMWR. 2013 June 28;62(2).

(3) Public Health Act Communicable Disease Regulation. BC Reg. 4/83. List of reportable diseases. British Columbia Health Act Communicable Disease Regulation [Internet]. http://www.bclaws.ca/Recon/document//D/freeside/12_4_83.

(4) Swinkels HM, Kuo M, Embree G, Fraser Health Environmental Health Investigation Team, Andonov A, Henry B, Buxton JA. Hepatitis A outbreak in British Columbia, Canada: The roles of established surveillance, consumer loyalty cards and collaboration, February to May 2012. Eurosurveillance. 2014;19(18).

(5) Taylor J, Galanis E, Wilcott L, Hoang L, Stone J, Ekkert J, Quibell D, Huddleston M, McCormick R, Whitfield Y, Adhikari B, Grant CC, Sharma D, Salmonella Chester Outbreak Investigation Team. An outbreak of Salmonella Chester infection in Canada: Rare serotype, uncommon exposures and unusual population demographic facilitate rapid identification of food vehicle. J Food Prot. 012;75(4):738-742.

(6) McIntyre L, Galanis E, Mykytczuk O, Buenaventura E, Wong J, Prystajeky N, Ritson M, Stone J, Moreau D, Youssef A, Outbreak Investigation Team. Multiple clusters of norovirus among shellfish consumers linked to symptomatic oyster harvesters. J Food Prot. 2012;75(9):1715-1720.

(7) Taylor M, Mclntyre L, Ritson M, Stone J, Bronson R, Bitzikos O, Rourke W, Galanis E, Outbreak Investigation Team. Outbreak of diarrhetic shellfish poisoning associated with mussels, British Columbia, Canada. Mar. Drugs. 2013;11(1):669-1676.

(8) Taylor M, Leslie M, Ritson M, Stone J, Cox W, Hoang L, Galanis E, Outbreak Investigation Team. Investigation of the concurrent emergence of Salmonella enteritidis in humans and poultry in British Columbia, Canada, 20082010. Zoonoses Public Health. 2012;59(8):584-592.

(9) King N, Lake R, Campbell D. Source attribution of nontyphoid salmonellosis in New Zealand using outbreak surveillance data. J Food Prot. 2011;74(3):438-445.

(10) European Food Safety Authority, European Centre for Disease Prevention and Control. The European Union summary report on trends and sources of zoonoses, zoonotic agents and food-borne outbreaks in 2013 [Internet]. Parma, Italy: EFSA; 2015.

http://ecdc.europa.eu/en/publications/Publications/EU-summary-report-trends-sources-zoonoses-2013.pdf.

(11) Taylor M, Galanis E. Establishing criteria to initiate enteric outbreak investigations in British Columbia. Can Commun Dis Rep 2014;40(S-1). (Disponible en français : http://www.phac-aspc.gc.ca/publicat/ccdrrmtc/14vol40/dr-rm40s-1/index-fra.php)

(12) Gaulin C, Currie A, Gravel G, Hamel M, LeBlanc MA, Ramsay D, Bekal S. Summary of 11 years of enteric outbreak investigation and criteria to initiate an investigation, Province of Quebec, 2002 through 2012. J Food Prot. 2014;9:1448-1648.

(13) Berger CN, Sodha SV, Shaw RK, Griffin PM, Pink D, Hand P, Frankel G. Fresh fruit and vegetables as vehicles for the transmission of human pathogens. Environ Microbiol. 2010;12(9):2385-2397.

(14) Lynch MF, Tauxe RV, Hedberg CW. The growing burden of foodborne outbreaks due to contaminated fresh produce: Risks and opportunities. Epidemiol Infect. 2009;137(3):307-315.

(15) Sivapalaingam S, Friedman CR, Cohen L, Tauxe RV. Fresh produce: A growing cause of outbreaks of foodborne illness in the United States, 1973 through 1997. J Food Prot. 2004;67(10):2342-2352.

(16) Butler A, Pintar K, Thomas MK. Estimating the relative role of various subcategories of food, water and animal contact transmission of 28 enteric diseases in Canada. Forthcoming 2015.

(17) Davidson VJ, Ravel A, Nguyen TN, Fazil A, Ruzante JM. Food specific attribution of selected gastrointestinal illnesses: Estimates from a Canadian Expert Elicitation Survey. Foodborne Pathog Dis. 2011;8(9):983-995.

(18) Ravel A, Davidson VJ, Ruzante JM, Fazil A. Foodborne proportion of gastrointestinal illness: Estimates from a Canadian expert elicitation survey. Foodborne Pathog Dis. 2010;7:1463-1472.

(19) Pires SM, Vieira AR, Hald T, Cole D. Source attribution of human salmonellosis: An overview of methods and estimates. Foodborne Pathog Dis. 2014;1:667-676.

(20) US Food and Drug Administration. FDA, federal partners develop improved method for attributing foodborne illness: Constituent update [Internet]. Washington DC: USFDA; Feb 24, 2015. http://www.fda.gov/Food/NewsEvents/ConstituentUpdates/ucm435256.htm. 